\title{
Agôn
}

Revue des arts de la scène

5 | 2012

L'entrée en scène

\section{L'entrée en scène de la Comtesse dans Les Noces de Figaro : contrainte dramaturgique ou levier de sens?}

Catherine Ailloud-Nicolas

\section{CpenEdition \\ Journals}

Édition électronique

URL : http://journals.openedition.org/agon/2452

DOI : 10.4000/agon.2452

ISSN : 1961-8581

Éditeur

Association Agôn

Référence électronique

Catherine Ailloud-Nicolas, «L'entrée en scène de la Comtesse dans Les Noces de Figaro : contrainte dramaturgique ou levier de sens ? », Agôn [En ligne], 5 | 2012, mis en ligne le 25 janvier 2013, consulté le 06 mai 2019. URL : http://journals.openedition.org/agon/2452 ; DOI : 10.4000/agon.2452

Ce document a été généré automatiquement le 6 mai 2019.

Association Agôn et les auteurs des articles 


\title{
L'entrée en scène de la Comtesse dans Les Noces de Figaro: contrainte dramaturgique ou levier de sens?
}

\author{
Catherine Ailloud-Nicolas
}

1 Face à un opéra, le metteur en scène de théâtre peut avoir l'impression que la question de l'entrée en scène se pose de la même façon que dans son art de référence. Faire entrer un chanteur en scène, c'est en effet, comme pour l'acteur, poser la question des frontières entre un hors-scène et un espace à vue, espace qui est lui-même le produit de la superposition entre le fictionnel et la réalité construite par la scénographie. C'est marquer aussi le début d'une présence dans un temps déterminé qui s'inscrit entre une entrée et une sortie.

2 Mais l'opéra est régi par des contraintes spécifiques. L'entrée en scène est en effet liée au chant si étroitement que, jusqu'à il y a peu de temps, les usages faisaient que le chanteur entrait uniquement quand il devait chanter ${ }^{1}$. En outre, il fallait que cette entrée soit peu ou prou dramatisée, mise en valeur, afin que soit respectée la hiérarchie entre personnages principaux et personnages secondaires, ou bien entre chanteurs pourvus d'un rôle et membre du chœur. Le regard du spectateur était habilement orienté vers le héros pour repérer sans aucune ambiguité le divo ou la diva ${ }^{2}$. À ces codes opératiques d'une certaine époque de la mise en scène, s'ajoute l'impression souvent ressentie à la lecture des livrets que la justification dramaturgique de l'entrée en scène, héritage au théâtre de toute une réflexion sur les liaisons des scènes, est moins nécessaire à l'opéra avec parfois des cas extrêmes où entrées et sorties peinent à trouver une raison autre que celle du développement formel en solos, duos et ensembles ${ }^{3}$.

Un cas particulier d'entrée en scène est celui où un personnage se présente seul sur le plateau pour chanter4. On ne s'intéressera pas aux débuts d'acte qui ne présentent pas de difficultés particulières ${ }^{5}$. L'utilisation du rideau peut à chaque fois donner l'illusion que le personnage est déjà là. Il est alors saisi dans une action déjà commencée. La convention se trouve contournée ou effacée. En revanche, tel n'est pas le cas quand la scène s'insère dans la structure interne de l'acte. L'entrée en scène du personnage se présente alors 
comme une contrainte forte pour le metteur en scène. Tel est le cas de l'air de la Comtesse dans l'acte III des Noces de Figaro. C'est lui qui sera l'objet de cette étude. En effet, en participant comme dramaturge à la mise en scène commandée à Richard Brunel ${ }^{6}$ par le Festival d'Art Lyrique d'Aix en Provence en 2012, j'ai pu mesurer l'importance de ce moment particulier autant comme problème ponctuel à régler que comme moyen d'interroger la globalité de l'acte. Afin de rendre compte de la réflexion qui a été conduite avec le metteur en scène, je calquerai le plan de cet article sur les étapes telles qu'elles ont été conduites dans la réalité du travail préparatoire, très en amont du début des répétitions. Je ferai dans un premier temps une analyse des problèmes dramaturgiques posés par cette entrée en scène. Dans un second temps, je comparerai différentes mises en scène ${ }^{7}$ en étudiant comment elles ont envisagé cette question et proposé des solutions. Enfin, je décrirai plus précisément les choix opérés par Richard Brunel afin de montrer comment une entrée en scène problématique peut devenir le noyau dramaturgique d'une proposition scénique.

\section{L'entrée en scène de la Comtesse : problèmes dramaturgiques}

Le premier problème dramaturgique concerne l'espace. Toute entrée en scène pose en effet une question principale : où entre le personnage? Et des questions périphériques : d'où vient-il ? Où va-t-il ?

L'acte III des Noces se situe dans un espace à la fois défini et mouvant. La didascalie initiale de l'Avant-scène opéra $a^{8}$ précise : « une salle somptueuse, avec deux trônes, et préparée pour la fête nuptiale. » C'est donc un espace public caractérisé par la fonction qu'il aura au final, c'est-à-dire une salle dans laquelle on se marie. Néanmoins, pendant tout le reste de l'acte, cette fonction est en quelque sorte effacée par tous les efforts que fait le Comte pour que justement le mariage ne puisse pas exister. Reste que les scènes de groupe attestent la dimension sociale de l'espace. Il est somme toute assez naturel que se retrouvent là Curzio, Marcellina, Bartolo, Figaro et Susanna car le Comte les reçoit pour régler des affaires judiciaires qui les concernent. En revanche, il est plus étonnant de voir se dérouler dans le même endroit des entretiens dont le caractère secret est incontestable. Ils n'ont pas pour vocation d'être écoutés par un tiers et il serait même dangereux qu'ils le soient. C'est le cas par exemple de la scène de la lettre entre la Comtesse et Susanna (scène 10) mais c'est aussi le cas du duo entre Cherubino et Barbarina (scène 7) dans lequel est évoqué le projet du déguisement du jeune page en fille. L'espace est donc aussi problématique que l'antichambre des tragédies du XVII siècle. Il est en quelque sorte à dramaturgie variable : de temps en temps défini par la thématique de la scène ${ }^{9}$, de temps en temps tendant vers une certaine forme de neutralité.

De plus, curieusement, dans cet espace mouvant, les scènes se succèdent en assumant le plateau vide. C'est le cas par exemple dans l'enchainement des scènes $6^{10}, 7,9$ et 10 . À chaque fois se déroule une scène courte avec des personnages qui quittent le plateau pour laisser la place à d'autres qui entrent sans les avoir vus. Il n'y a aucune liaison de scènes prise en charge par le texte. Dans le livret publié par L'Avant-scène opéra, une indication didascalique précise à chaque fin de scène qu'une sortie a lieu ${ }^{11}$. En revanche, l'entrée est 
en quelque sorte court-circuitée. On indique le nom du ou des personnages présents dans la scène comme s'il y avait difficulté à rendre en compte des modalités de leur arrivée.

7 À la convention d'un espace unique, s'ajoute donc la convention d'entrées en scène sur un plateau nécessairement vide. Cet enchaînement de scènes donne à l'espace actuel une apparence de lieu de passage ${ }^{12}$ qui lui permet de s'adapter aux enjeux représentés, principalement ceux concernant le Comte. Il n'acquiert une fonction stabilisée qu'à la fin de l'acte quand le mariage a lieu.

Dans ces conditions, l'entrée en scène de la Comtesse est très problématique car on se demande ce qu'elle vient faire dans un espace qui est avant tout celui de l'intrigue du mariage de Susanna et qui est dominé par son époux. De fait, l'incongruité de sa présence a conduit da Ponte à la justifier au début de l'air : «E Susanna non vien! sono ansïosa di saper come il Conte accolse la proposta ${ }^{13}$ : alquanto ardito il progetto mi par, e ad uno sposo si vivace e geloso... $»^{14}$. C'est l'impatience qui fait venir la Comtesse alors qu'en toute logique elle devrait attendre Susanna chez elle. Il y a donc là une logique d'ordre psychologique.

Néanmoins, cette logique ne parvient pas à effacer une incohérence: celle de l'enchaînement des faits. En effet, dans le sextuor de la scène 5, Susanna ${ }^{15}$ arrive avec deux mille piastres qu'elle remet au Comte pour payer la liberté de Figaro. Or, la seule personne susceptible de lui avoir remis cette somme est la Comtesse. Dans ces conditions, il faut admettre que la Comtesse a vu Susanna hors-scène, ce qui détruit la justification du récitatif. Et quand elle la retrouve à la scène 10, la question qu'elle pose à Susanna porte sur les réactions du Comte face au retournement de situation et non sur le rendez-vous dans le jardin. La logique des événements est donc plus vraisemblablement :

\begin{tabular}{|c|c|c|c|c|}
\hline Scène & $\begin{array}{l}\text { La } r \text { Comtesse } \\
\text { demande à } \\
\text { Susanna de donner } \\
\text { un rendez-vous au } \\
\text { Comte (scène 2) }\end{array}$ & $\begin{array}{l}\text { La Comtesse } \\
\text { s'impatiente car } \\
\text { Susanna ne revient } \\
\text { pas (scène 8) }\end{array}$ & & 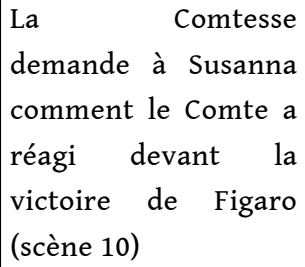 \\
\hline $\begin{array}{l}\text { Hors- } \\
\text { scène }\end{array}$ & & & $\begin{array}{l}\text { La Comtesse donne } \\
\text { à Susanna l'argent } \\
\text { dû à Marcellina } \\
\text { (pendant la scène } \\
\text { 5) }\end{array}$ & \\
\hline
\end{tabular}

L'entrée en scène dans la huitième scène fait apparaître une rupture dans l'enchaînement des événements. Cette incohérence dramaturgique vient en réalité d'un problème purement technique que rappelle Jean de Solliers dans l'Avant-scène opéra: "Car ce qui a motivé l'ordre actuel des scènes, c'est, comme au final du $2^{\text {ème }}$ acte, le fait que le même chanteur tenait à l'origine les rôles de Bartolo et d'Antonio, et qu'il n'aurait pas eu le temps de changer de costume pendant la courte scène VII, si on n'avait pas intercalé la scène VIII (l'air de la Comtesse) $»^{16}$. Les chefs d'orchestre et les metteurs en scène ont donc aujourd'hui le choix de respecter l'ordre de la partition ou de privilégier un enchaînement des scènes qui donne une plus grande validité au début du récitatif. Le choix a évidemment des conséquences sur la façon dont l'entrée de la Comtesse va 
apparaitre : étape dans le déroulement de l'intrigue ou au contraire parenthèse lyrique dans un acte dominé par l'action.

\section{De la dramaturgie à la mise en scène : quelques solutions}

11 Les metteurs en scène doivent donc proposer des réponses aux questions posées par la dramaturgie. On peut les résumer ainsi : où la Comtesse entre-t-elle en scène ? Son entrée en scène est-elle rendue légitime par l'ordre des scènes ? Comment cette entrée en scène est-elle traitée c'est-à-dire comment sont organisées les transitions entre les scènes ? Comment l'entrée en scène induit-elle un traitement de l'air lui-même?

Les sept mises en scène étudiées offrent un panel intéressant. Entre le classicisme flamboyant de Strehler et les audaces de Marthaler et Sellars, se déclinent des propositions qui reposent toutes sur une réflexion dramaturgique forte ${ }^{17}$.

Le premier constat que l'on peut faire est que, à l'exception de Jean-Louis Martinoty ${ }^{18}$, tous les artistes respectent l'ordre des scènes de la partition. Le metteur en scène français a procédé, quant à lui, à des modifications très importantes :

\begin{tabular}{|l|l|l|l|}
\hline \multicolumn{2}{|l|}{ Ordre de la partition } & \multicolumn{2}{l|}{ Ordre adopté par Martinoty } \\
\hline Scène 4 & Monologue du Comte & Scène 4 & Monologue du Comte \\
\hline Scène 5 & Résultat du procès & Scène 7 & Barbarina/Cherubino \\
\hline Scène 6 & $\begin{array}{l}\text { Quatuor : Susanna, Figaro, } \\
\text { Marcellina, Bartolo }\end{array}$ & Scène 8 & Comtesse \\
\hline Scène 7 & Barbarina/Cherubino & Scène 5 & Résultat du procès \\
\hline Scène 8 & Comtesse & Scène 6 & $\begin{array}{l}\text { Quatuor : } \\
\text { Marcellina, Bartolo }\end{array}$ \\
\hline Scène 9 & Antonio/Comte & Scène 9 & Antonio/Comte \\
\hline $\begin{array}{l}\text { Scène } \\
10\end{array}$ & Comtesse/Susannaro, & $\begin{array}{l}\text { Scène } \\
10\end{array}$ & Comtesse/Susanna \\
\hline
\end{tabular}

Jean-Louis Martinoty résout donc, par cette modification de l'ordre, le problème dramaturgique que nous avons souligné. Il accentue la logique de l'enchaînement des faits ${ }^{19}$. La scène entre Barbarina et Cherubino et celle de la Comtesse apparaissent comme des parenthèses intimes et secondaires par rapport à l'intrigue principale.

La conclusion que l'on peut tirer de ce premier constat est que dans leur grande majorité, les metteurs en scène ne sont pas gênés par l'énigme dramaturgique de l'entrée en scène de la Comtesse. Ils respectent l'ordre imposé, ils s'en accommodent. Comment l'entrée en scène de la Comtesse s'inscrit-elle alors dans un fonctionnement plus global?

La première question que nous avons posée est celle de l'espace dans lequel se fait cette entrée en scène. Il est frappant de constater que les metteurs en scène, dans leur grande 
majorité, n'ont pas souhaité une scénographie qui anticipe sur les noces à venir. Seul Sven-Eric Bechtolf ${ }^{20}$ et son scénographe Rolf Glittenberg installent une salle de mariage : des rangées de chaises recouvertes de tissu blanc font face à une sorte de petit théâtre, une scène placée au fond du plateau. Les autres metteurs en scène construisent un espace qui n'est pas d'emblée caractérisé par la fonction qu'il occupe à la fin de l'acte. Et les choix opérés sont soit une autre détermination, soit une certaine forme d'abstraction. Dans le premier cas, on peut citer Strehler ${ }^{21}$. Avec son scénographe Ezio Frigerio, il place l'ensemble de l'acte III dans un gigantesque salon de musique où trône un piano à queue. L'espace signe donc avant tout l'activité sociale aristocratique et en même temps la mise en abyme potentielle avec l'opéra lui-même puisque l'on peut y chanter. En tout cas, le mariage a lieu dans un lieu qui n'est pas prévu pour cela. Partant, l'entrée en scène de la Comtesse n'a aucun caractère incongru puisque le Comte n'a pas l'exclusivité de cet espace. C'est le cas aussi dans la mise en scène de Sellars ${ }^{22}$. L'acte III se situe dans le salon d'un appartement de la Trump Tower ${ }^{23} \mathrm{de}$ New York. D'immenses vitres révèlent le soir qui tombe sur la ville. Un escalier donne sur une sorte de mezzanine, créant ainsi deux plans, deux scènes possibles. Là encore, le salon ne dit pas le mariage et même si deux fauteuils à hauts dossiers font référence aux deux trônes de la didascalie du livret, ils sont avant tout ici les créations d'un designer pour l'appartement du couple Almaviva.

Les autres propositions jouent plutôt sur une forme d'abstraction. Certes Anna Viebrock, la scénographe de Christoph Marthaler ${ }^{24}$, semble représenter un bureau d'état-civil assez réaliste en arrière-plan. Cependant, la scène montre aussi à jardin et à cour des vitrines avec des costumes de mariés, et au centre un espace qui pourrait être celui d'un appartement avec un bar, un fauteuil (le fameux fauteuil de l'acte I). Il y a là une sorte d'esthétique de collage qui, à partir d'éléments imitant des lieux réels finit, par le biais de l'effet de juxtaposition, par créer un espace symbolique hétérogène. Les personnages peuvent entrer dans cet espace sans qu'une justification soit nécessaire car il n'appartient à personne, il est par essence pluriel, multiple.

18 Les trois autres mises en scène de notre corpus vont plus loin dans l'indétermination ou l'abstraction. Ainsi Claus Guth ${ }^{25}$ et son scénographe Christian Schmidt placent l'ensemble de l'acte III dans un gigantesque escalier. La scène en est un palier à partir duquel une volée de marches monte et une autre descend. Les entrées en scène se font directement sur le palier, en haut des marches par des portes ou bien encore par l'escalier du bas. Cette dernière solution provoque une entrée en scène progressive, la tête apparaissant d'abord et le corps ensuite. Le grand escalier donne une impression de très grande abstraction. Il apparaît, en outre, comme le lieu de passage par essence. Les personnages peuvent rester sur le palier un moment mais ils ont d'abord comme vocation d'aller ailleurs. L'escalier signe la transition comme il peut aussi signer les rapports hiérarchiques: les nobles plus naturellement vont vers le haut, les autres personnages arrivent par le bas ou par les portes latérales. Cet espace est donné à voir dans son caractère monumental, il n'évolue pas. Ce n'est pas le cas des propositions de Martinoty et de Sivadier mais pour des raisons différentes. Martinoty et son scénographe Hans Schavernoch déclinent d'acte en acte le même principe : l'espace est structuré par un ensemble de tableaux, superposés ou placés les uns à côté des autres et qui indiquent une spécificité thématique de l'acte. Celle de l'acte III évoque les cabinets de curiosité du XVIII ème siècle, idée renforcée par la présence à jardin d'une table recouverte d'une nappe et de divers objets comme un sablier, des papillons sous verre et un crâne. Cet espace peut être modifié car les toiles sont mobiles. Ainsi, à la scène 10, quelques unes d'entre elles sont 
soulevées et dévoilent la Comtesse en conciliabule avec Susanna, Figaro, Marcellina et Bartolo. Mais surtout cet ensemble de tableaux permet de servir de passage aux divers personnages pour aller de jardin à cour, ou pour surgir du fond vers l'avant-scène. Il fonctionne en réalité comme une sorte de coulisse.

L'espace est sans doute plus lié au Comte qu'à la Comtesse ${ }^{26}$, le cabinet des curiosités étant une activité plus masculine que féminine, à l'époque. Mais il se révèle relativement abstrait car au bout du compte, ce qu'il dit avant tout c'est la multiplication ${ }^{27}$ et la cachette. Par ailleurs, le fait que le procédé scénographique se répète d'acte en acte lui enlève de la détermination pour le faire tendre vers l'abstraction. Plus qu'un moyen de désigner, il y a là avant tout un principe de déclinaison qui inscrit chaque proposition dans une cohérence globale.

Jean-François Sivadier ${ }^{28}$ va plus loin dans cette voie. En effet, il a un univers de référence : le théâtre. Il applique à chaque production une sorte de signature scénographique à partir de quelques invariants. Parmi ceux-ci, on trouve les toiles peintes de Jean-Baptiste Marot, le décor en continuel mouvement d'Alexandre de Dardel, décor destiné à donner l'illusion d'une machinerie découverte, d'un entre-deux, d'un intermédiaire entre le plateau et la coulisse. Sivadier ne construit pas une scénographie complètement et uniquement en fonction d'une dramaturgie spécifique. Il adapte aussi les principes qui sont toujours les siens, à la pièce, et à l'intérieur de l'opéra à chaque acte. On trouve dans l'espace de l'acte III, des traces de l'acte précédent: le lit de la Comtesse, des modules avec des portes, un petit escalier qui a servi à l'entrée d'Antonio dans l'acte II et que l'on a poussé. L'espace chaotique, désordonné, dit le refus du décor mimétique ainsi que le désir d'affirmer une théâtralité triomphante, entre revendication du bricolé et fonctionnalité. Et c'est dans son rattachement à une esthétique et des principes de théâtre qui dépassent le cadre précis d'un opéra spécifique qu'il trouve une certaine abstraction. L'entrée en scène de la Comtesse est donc traitée comme un élément à l'intérieur d'un système général dans lequel on fait semblant d'adapter, dans l'urgence et au vu de tous, l'espace à ce que l'on joue. Il n'y a aucune logique topographique, il y a du théâtre qui s'adapte à du théâtre.

21 Que conclure de cette première série d'observations? Parmi les mises en scène qui ne caractérisent pas l'espace comme étant celui du mariage à venir, il y a effacement de l'identité du lieu. La Comtesse peut légitimement entrer dans ces espaces-là car ils lui appartiennent autant qu'au Comte ou bien parce qu'ils ne lui appartiennent pas plus qu'au Comte. L'incongruité éventuelle de sa présence à cet endroit précis est en quelque sorte gommée. Dans le seul cas de notre corpus qui représente une salle préparée pour un mariage, l'entrée de la Comtesse est très clairement associée à ce mariage. Elle entre par la scène placée au fond en portant un bouquet de fleurs qu'elle vient disposer pour les noces. Elle enlève un drap qui recouvre des fauteuils. Elle se conduit donc en hôtesse tout en manifestant clairement qu'elle contribue à sa façon à la préparation du mariage. Elle agit sur l'espace pour en renforcer la fonction. La justification de sa présence est donc scénique, étroitement liée à l'espace.

Dans tous les cas, l'espace étant unique, l'entrée en scène de la Comtesse se trouve finalement en résonance avec toutes celles de l'acte. On ne s'étonnera pas, dans ces conditions, que la succession des entrées en scène nécessite un nombre de portes très important. S'ajoutent à ces portes des sous-espaces qui permettent aux personnages de ne pas se rencontrer comme la mezzanine de Sellars, la scène du fond de Bechtolf, l'escalier de Guth, le bureau d'état-civil de Marthaler. L'espace unique ne se modifie pas, il se 
structure en sous-espaces ou il s'ouvre sur des ailleurs multiples. Il est défini par sa segmentation ou sa porosité.

Cette variété des possibles pensée par la scénographie va de pair avec des choix divers sur le traitement des entrées en scène: leur succession est renforcée, les liens topographiques sont organisés ou bien les entrées et sorties de scènes sont superposées. Strehler souligne la succession des entrées et des sorties de scène plutôt que de la gommer. Il fait entrer la Comtesse dans un espace vidé de ces précédents occupants. Elle s'avance vers le public, chante son air, puis elle repart par une porte latérale à cour ${ }^{29}$. Après un bref moment, Antonio et le Comte entreront par une porte latérale à jardin. Il y a donc respect de la partition et risque assumé du plateau vide. Un bref instant, ce salon de musique immense se trouve déserté avant d'être investi par de nouveaux personnages qui y passent après une rapide station. Sivadier, conscient de la convention, va plus loin, il la met en scène. En effet, chaque entrée en scène est précédée d'un changement de décor par un machiniste et par une sonnerie comme celle qui appelle en coulisse le chanteur à rejoindre le plateau. Il se présente pendant un silence, il se place et il se met à chanter. L'entrée en scène du personnage est donc précédée en quelque sorte par l'entrée du chanteur. L'entrée de la Comtesse obéit à ce code. Une convention s'ajoute à une autre convention pour la souligner et la dénoncer.

Marthaler joue aussi de la convention mais en portant son attention sur les récitatifs. En effet, ceux-ci sont assumés scéniquement par Jürg Kienberger. Mais le musicien ne se contente pas d'accompagner les récitatifs avec des instruments étranges, il joue un rôle essentiel dans les entrées et les sorties de scène. À la fin de la scène 6, il chante un yodel si surprenant que Figaro, étonné, revient sur ses pas puis il va vers le fond de la scène où il prend des bouteilles de bière. Il alterne les moments où il boit et ceux où il souffle dans les bouteilles pour soutenir la scène entre Barbarina et Cherubino. Pour l'entrée en scène de la Comtesse, en revanche, il sort et laisse la place à l'orchestre puis il revient après l'air. Les entrées en scène des personnages sont donc doublées par l'entrée en scène de ce musicien un peu particulier qui fait un parcours poétique sur le plateau ${ }^{30}$.

Les metteurs en scène compensent parfois l'artificialité de la succession d'entrées et de sorties par la mise en place d'une logique topographique qui fait que chaque entrée en scène est en réalité reliée à une sortie de scène ou/et à une entrée en scène antérieures ou postérieures. Ainsi Strehler fait sortir la Comtesse par la porte qu'elle empruntera pour revenir à la scène 10 avec Susanna ${ }^{31}$. La topographie vient au secours de la dramaturgie. Enfin, la dernière solution pour lutter contre la convention est de créer des superpositions entre les scènes qui évitent la succession des entrées et des sorties. Pour ce faire, sont utilisés deux procédés. Le premier résulte des potentialités offertes par l'espace. Ainsi, dans la mise en scène de Sellars, la mezzanine permet la coexistence de deux plans : Barbarina et Cherubino ne quittent pas l'espace à la fin de leur scène, ils s'embrassent en bas pendant que la Comtesse entre sur la mezzanine. La scène 7 se poursuit donc pendant que la scène 8 commence. Le tuilage atténue la convention. Bechtolf procède systématiquement ainsi de façon à créer des enchaînements fluides. Il utilise, pour ce faire, la petite scène qui se trouve au fond de son décor. Ainsi, pendant la scène 6, alors que Figaro, Susanna, Marcellina et Bartolo sont à l'avant-scène, on voit au fond passer Cherubino et Barbarina qui font les fous avant de venir se cacher sous des fauteuils. Quand le quatuor est sorti, ils réapparaissent. Barbarina remonte sa culotte et Cherubino se reboutonne, laissant imaginer leur activité pendant la scène précédente. Ils sortent par une porte à jardin pendant que la Comtesse entre par la scène. Elle chante en 
avançant progressivement vers la face. À la fin de l'air, Susanna la rejoint. Elles se cachent toutes deux derrière les fauteuils, à l'avant-scène, pendant que le Comte et Antonio passent au fond. La scène 10 prolonge donc logiquement l'air de la Comtesse, dans la continuité de la présence scénique des corps. Cette fois, c'est Susanna qui rejoint la Comtesse. Dans ces deux exemples, la convention est, autant que faire ce peu, atténuée ou effacée ${ }^{32}$. Le tuilage a une autre conséquence très intéressante : il y a des spectateurs ou des auditeurs à des scènes supposément intimes. Le dispositif des deux étages créé par Sellars apporte une grande ambiguïté. En effet, on ne sait pas si la Comtesse voit les deux jeunes amoureux en train de s'embrasser ni si ceux-ci l'entendent. Cela fonctionne plutôt comme deux plans cinématographiques, comme deux unités parallèles. En revanche, Jean-François Sivadier assume le fait que le jeune couple entend l'air de la Comtesse ${ }^{33}$. À la fin du duo, un machiniste réorganise l'espace. Une grande table est enlevée, des blocs de porte sont replacés. La Comtesse entre par le fond, elle ouvre un bloc porte et le referme et s'avance vers le public. Les deux jeunes gens sont en retrait et dans une logique naturaliste, elle devrait les voir mais ici ce n'est pas le cas. Ils sont à terre, Cherubino a la tête sur les genoux de Barbarina. À la fin de l'air, ils se dirigent vers une porte en veillant à ne pas se faire remarquer. Barbarina sort. Cherubino revient sur ses pas et reproduisant un geste qu'il fait fréquemment dans l'opéra, il décoche une flèche en direction de la Comtesse. Les deux plans coexistent mais interagissent: l'air de la Comtesse existe pour d'autres qu'elle-même. Il ne perd pas son statut de monologue car il n'est pas adressé, mais il est entendu par des récepteurs additionnels : par le jeune couple dans un premier temps, puis dans un deuxième temps par Susanna, debout, sur le lit placé au fond de la scène. L'air concerne les autres personnages et le spectateur en a conscience.

L'entrée en scène dans un espace vide crée un focus sur le jeu de la chanteuse. La Comtesse, isolée ou solitaire, se retrouve dans la situation de l'acteur qui monologue. Elle doit motiver les bifurcations musicales par son seul jeu. Pour ce faire, les metteurs en scène choisissent deux voies principales. La première consiste à donner la première place au chant. Les changements dans la musique deviennent alors le reflet de modifications psychologiques. Le plus radical dans cette voie est sans doute Strehler. Trois mouvements sont apparents grâce surtout aux expressions du visage de la chanteuse. Tout d'abord, il y a un moment de plaisir devant le stratagème imaginé puis un moment de désarroi face à l'infidélité du Comte avant que soit retrouvé l'espoir à la fin de l'air. Ce découpage se retrouve avec des variantes chez Sellars par exemple. La Comtesse arrive sur la mezzanine, déjà sujette au trouble. Elle acquiert de l'énergie à la fin de l'air, mais c'est l'énergie du désespoir. On retrouve le même développement chez Marthaler. Tout ce qui est produit dans la scène l'est finalement dans des mouvements de pensée, ce qui accentue la dimension psychologique de la convention. Peu de metteurs en scène donnent des appuis de jeu à la chanteuse, en dehors de ces motivations intérieures. Parfois, des objets ont cette fonction. Ainsi, dans la mise en scène de Martinoty, la Comtesse commence à manipuler ce qui se trouve sur la table du cabinet de curiosité. Elle serre contre elle le sablier, puis elle touche par mégarde le crâne, ce qui la fait sursauter ${ }^{34}$. Dans les mises en scène de Bechtolf, Guth et Sivadier, la Comtesse joue avec des objets qui ont un rapport avec le mariage : robe, couronne, voile. Et chez Sivadier ce signe se superpose avec un signe extra fictionnel propre aux codes du metteur en scène : la Comtesse enlève sa perruque ${ }^{35}$. Ces appuis de jeu qui rattachent les mouvements de la musique à des actions de plateau soulignent tous néanmoins ce qui est dit. Ils n'apportent pas un autre éclairage sur l'air. Les objets liés à la noce de Susanna sont ainsi convoqués pour souligner 
la réflexion de la Comtesse sur son propre mariage. Ils renvoient de ce fait à une dimension psychologique car ils justifient des bifurcations de pensée et de musique.

L'entrée en scène de la Comtesse apparaît donc paradoxale quand elle est appréhendée par le plateau. Elle est un problème dramaturgique puisqu'elle est un marqueur d'incohérence. Elle devrait donc être l'objet d'une attention particulière. Or même dans des mises en scène très inventives comme celles de Marthaler, la scène est finalement traitée de façon assez classique ${ }^{36}$. C'est comme si les metteurs en scène s'inclinaient devant la musique, la laissaient passer au premier plan par rapport à la logique de l'action. De fait, même si la question du lieu est résolue, même si l'incongruité de l'entrée en scène est atténuée par le traitement de l'espace, ce qui se joue là est de l'ordre de la parenthèse psychologique. La Comtesse n'agit même plus sur le procès ${ }^{37}$. Elle est en dehors de l'action, réduite à la lamentation.

\section{L'entrée en scène de la Comtesse dans la mise en scène de Richard Brunel : un nœud dramaturgique}

Richard Brunel est un metteur en scène de théâtre passionné de musique. Il lui a toujours accordé une place importante dans ses spectacles: présence d'airs lyriques ${ }^{38}$ ou de musique classique, acteurs chantant ${ }^{39}$ ou chanteurs jouant ${ }^{40}$, collaboration avec le quatuor Debussy ${ }^{41}$. Il est entré en opéra grâce à une œuvre de Brecht, Celui qui dit oui, celui qui dit non et depuis lors il a été sollicité par le monde lyrique à de nombreuses reprises ${ }^{42}$. Ce qui intéresse au plus haut point Richard Brunel lorsqu'il aborde une œuvre musicale, c'est comment en révéler ou en accentuer la théâtralité. Cela signifie plusieurs choses : mettre en valeur la logique des événements et des situations, traquer les conventions pour les déjouer, aussi bien celles émanant du livret que celles concernant le jeu des chanteurs. L'enjeu est in fine de mettre le théâtre au service de la musique afin qu'elle en soit sublimée.

Dans le débat qui oppose encore aujourd'hui les partisans d'une mise en scène réduite au minimum et ceux d'une théâtralité affirmée jusqu'à la surinterprétation ${ }^{43}$, ce projet peut sembler utopique mais néanmoins c'est celui qui fédère toutes les mises en scène de Richard Brunel. Et bien qu'elles soient très différentes les unes des autres, elles ont des caractéristiques communes: parti pris très affirmé mais contenu dans les limites imposées par l'œuvre, effacement des conventions, jeu précis et débarrassé des clichés opératiques ${ }^{44}$. Pour ce faire, un long travail dramaturgique se fait en amont ${ }^{45}$. Celui sur Les Noces a duré environ un an et demi. La réflexion, dans ce type de production, se décompose en plusieurs étapes : pistes initiales, travail avec la scénographe, élaboration du projet de mise en scène ${ }^{46}$.

L'entrée en scène de la Comtesse s'est donc trouvée au croisement de plusieurs questionnements : la détermination des questions dramaturgiques globales et locales; la conception de l'espace, le traitement de la scène en soi et dans son rapport avec les autres scènes. Lors de l'étape initiale, le problème de la logique dramaturgique a été placé au premier plan. En effet, il paraissait important de montrer comment, dans cet acte, la Comtesse agit à la fois sur scène et hors-scène, parfois grâce à des intermédiaires et parfois directement. L'air de la Comtesse est un moment d'articulation dans ce cheminement. En effet, c'est le moment où la décision d'agir trouve sa motivation ${ }^{47}$. Par ailleurs, il était important d'affirmer l'enchaînement des événements : c'est parce que le 
verdict du procès de Figaro est posé, à savoir payer ou épouser Marcellina, que Susanna sort de scène pour aller demander de l'aide à la Comtesse qu'elle n'a pas vue depuis le début de l'acte.

L'ordre imposé par la partition a été modifié. L'enchaînement des scènes a donc été : monologue du Comte (4), Barbarina/Cherubino (7), air de la Comtesse (8), fin du procès (5), quatuor (6).

32 Cette logique des enchaînements des faits s'est trouvée confrontée à deux autres questions connexes. La première concerne l'espace et la seconde le parti pris global. Un des axes dramaturgiques forts, défini assez tôt dans le travail, concernait l'actualisation de l'opéra ${ }^{48}$. Il s'agissait de le ramener à notre époque mais en s'appuyant fermement sur ce qu'il dit. Ainsi puisque le Comte répète en permanence que c'est à lui de juger, Richard Brunel a décidé, avec sa scénographe Chantal Thomas, de le lier au monde judiciaire ${ }^{49}$ : il dirige un cabinet d'avocat ou de juge. L'acte I présente un lieu de travail avec une salle de réunion, et des portes qui mènent à des bureaux. L'acte III nous fait entrer dans un de ces bureaux. Nous sommes dans le même lieu mais vu selon une perspective différente.

Maquette de la scénographie des Noces de Figaro (2012) mis en scène par Richard Brunel

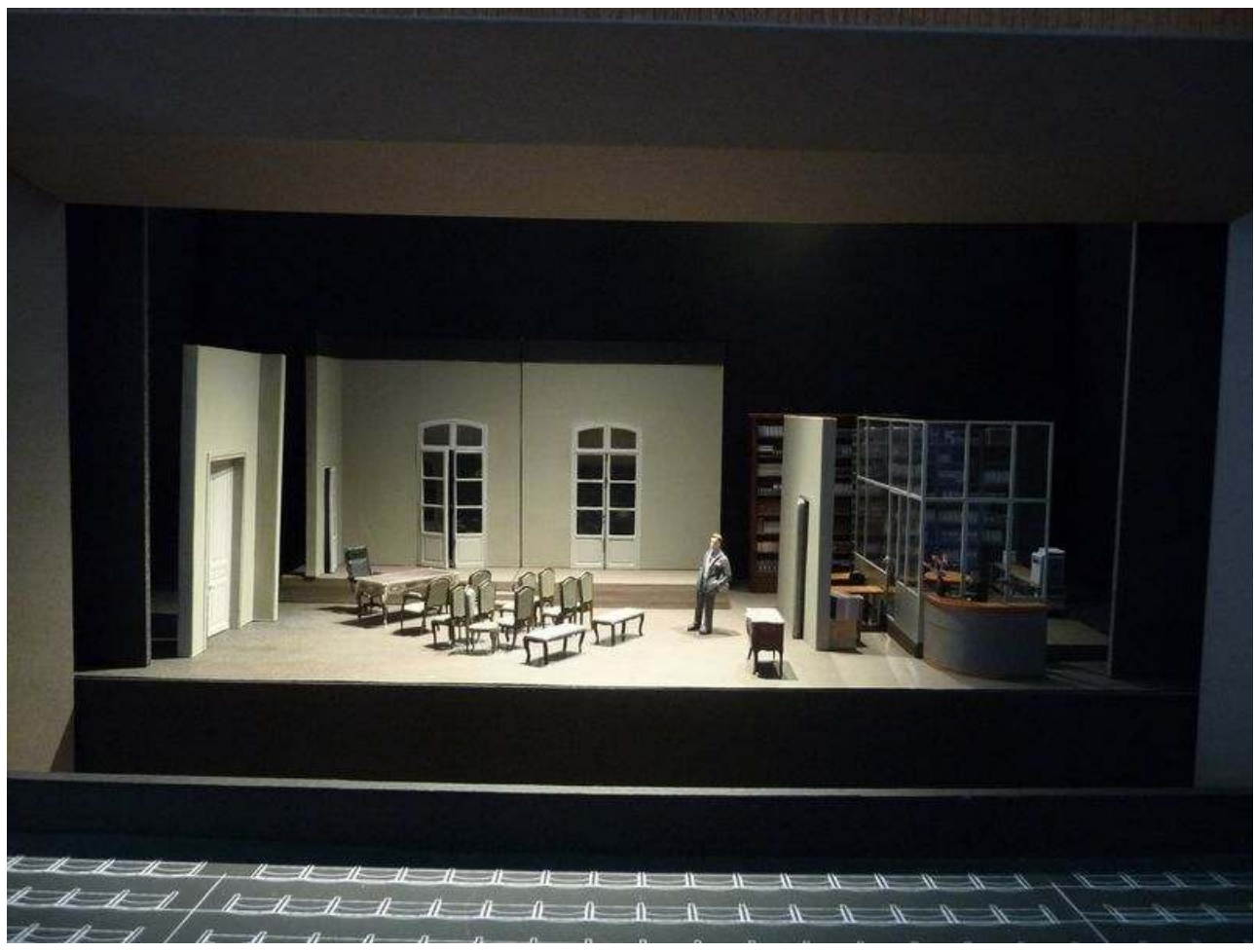

Chantal Thomas

Le déplacement de l'axe dans l'acte III structure l'espace en deux sous-espaces séparés par une porte : d'un côté, le bureau du Comte qui va pourvoir se métamorphoser selon que le personnage est avocat ou maire; et de l'autre côté le standard et la salle de réunion. Cette fragmentation permet de résoudre la question des enchaînements de scènes dans la mesure où il $\mathrm{y}$ a là deux sous-espaces qui peuvent être alternativement, selon les besoins, poreux ou étanches et qui permettent aussi le déroulement de scènes parallèles. 
Richard Brunel, Les Noces de Figaro (2012)

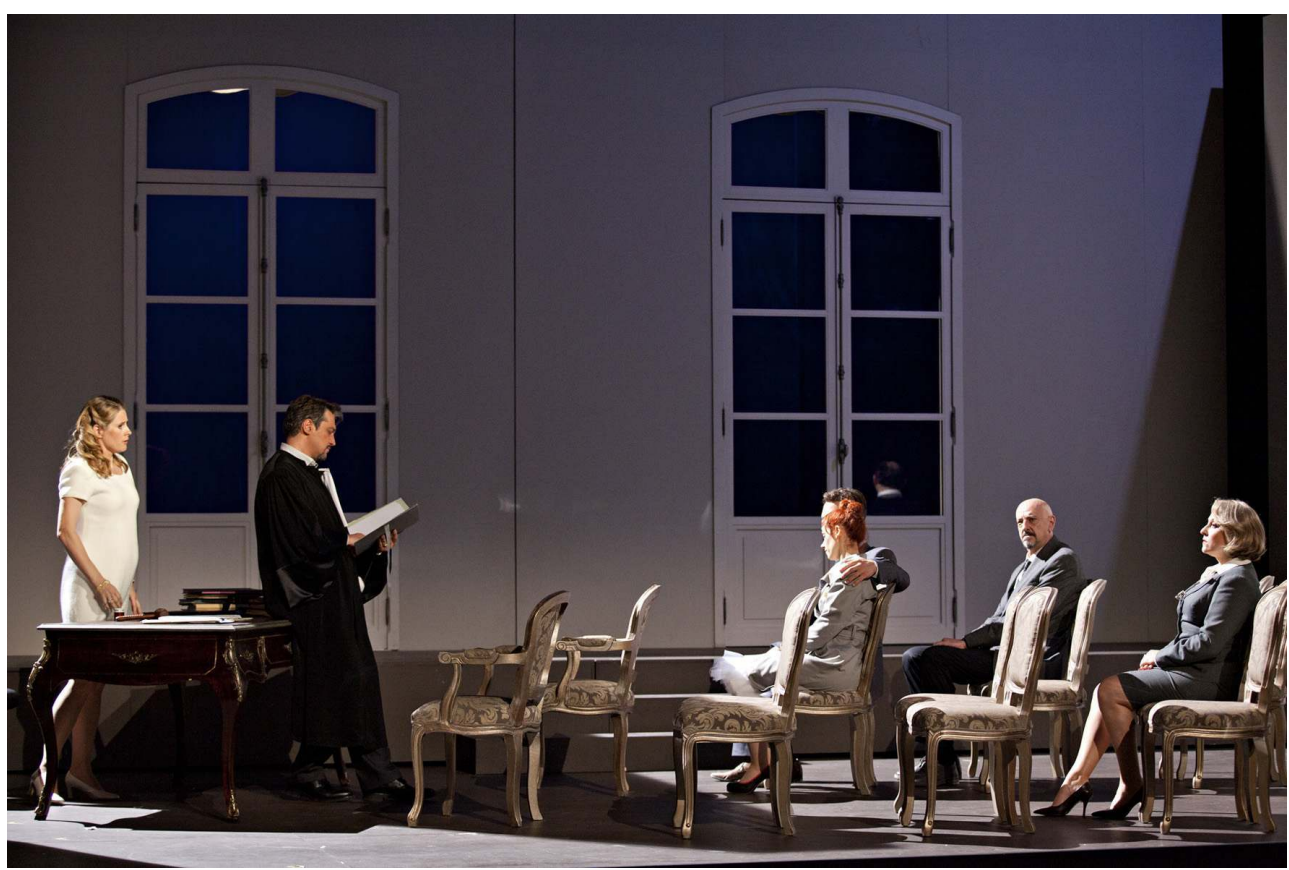

Jean-Louis Fernandez

Un deuxième parti pris lié au sens a conduit la réflexion. Dans l'opéra, le Comte ne cesse de dire qu'il va juger les affaires et il ne le fait jamais. Il est dédouané de cela par le fait que Curzio lui rend compte du résultat d'un procès qui s'est déroulé hors-scène. On voit bien là le résultat de l'autocensure opérée par da Ponte. La scène de procès, si importante dans la pièce de Beaumarchais, est supprimée. Dès lors, le Comte n'est pas pris en flagrant délit de confusion entre sa charge et ses sentiments. L'opéra édulcore la responsabilité du personnage. Réintégrer la scène du procès permettrait de redonner au Comte la place qui lui revient en accord avec tout ce qu'il dit dans l'opéra par ailleurs ${ }^{50}$. Jean-François Sivadier a tenté de le faire en insérant dans l'opéra une scène de théâtre, c'est-à-dire une scène muette sans musique. Cette solution est séduisante mais au prix d'une rupture problématique à la fois dans les codes de genre et les codes de jeu. La musique laisse la place au silence ${ }^{51}$. La solution adoptée par Richard Brunel est autre. Il s'agit de concilier plusieurs impératifs: rendre l'entrée en scène de la Comtesse cohérente dans le déroulement des événements et intégrer la scène du procès sans supprimer la musique. La solution est née de l'analyse du texte de l'air de la Comtesse. En effet après la justification de son entrée, elle se lance dans une sorte de plaidoirie qui est autant une défense de ses fautes ${ }^{52}$ qu'une accusation contre son mari ${ }^{53}$. Seule la deuxième partie de l'air apparait véritablement lyrique. L'entrée en scène de la Comtesse n'est donc pas forcément sur le mode du désespoir et de la déploration. Alors que se déroule le procès de Figaro, elle fait elle-même le procès du Comte. Mais ces deux procès ne se déroulent pas sur le même plan, l'un est un procès réel et l'autre est un procès imaginaire. 
Richard Brunel, Les Noces de Figaro (2012)

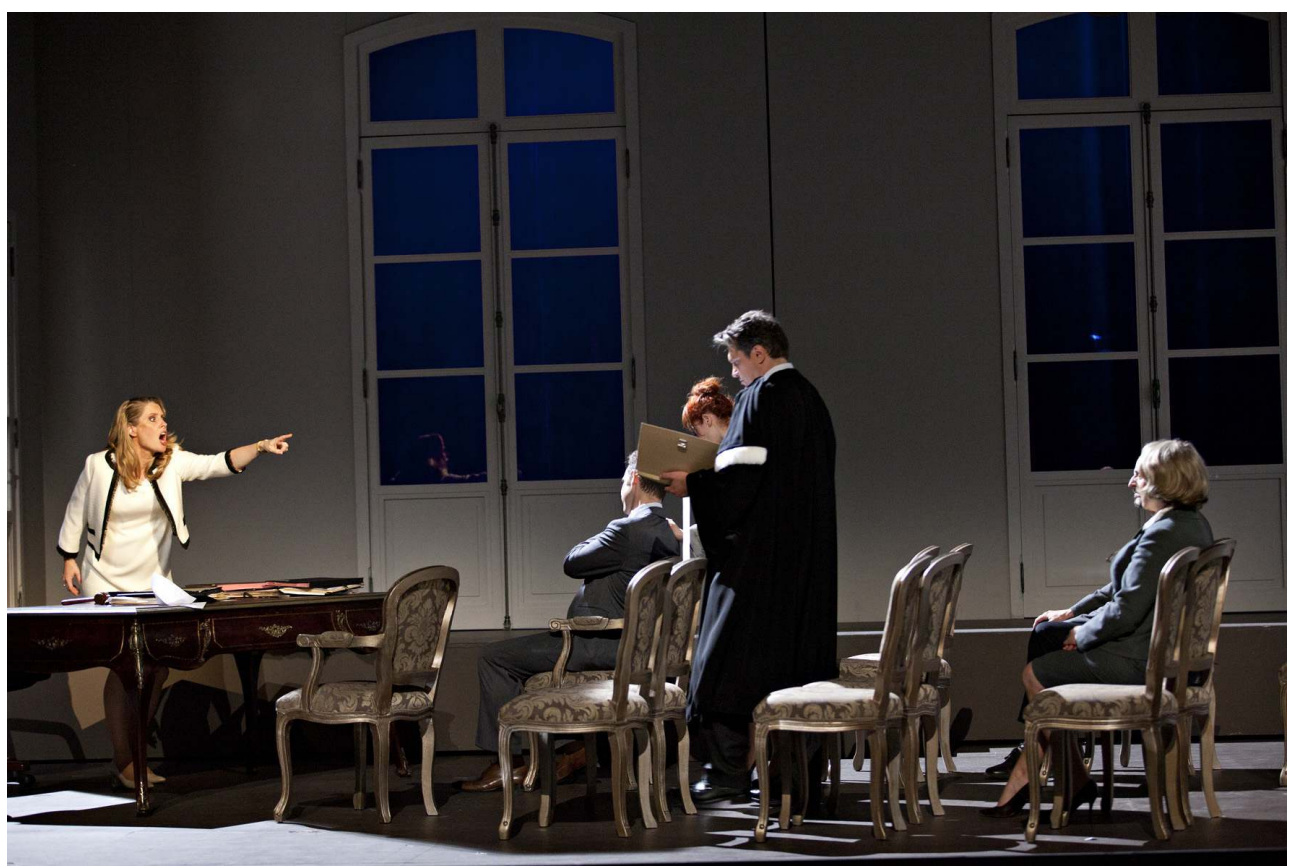

Jean-Louis Fernandez

La mise en scène rassemble toutes ces données. Le déroulé à partir de la scène 4 est le suivant. Le monologue du Comte est le marqueur d'une décision, celle de condamner Figaro $^{54}$. C'est le signal pour que s'installe, dans le bureau du Comte, une salle de procès. Pendant qu'il chante, le Comte donne, par des gestes, des instructions sur le placement des chaises. À la fin de son air, tous les personnages concernés par le procès s'assoient. Pendant cette installation, Cherubino rejoint Barbarina qui est restée dans son espace de standardiste. Ils quittent le plateau à cour au moment où la Comtesse fait son apparition à jardin. La suite est une superposition de deux actions, de deux temps et de deux espaces. Pendant que se déroule le procès de Figaro au ralenti, la Comtesse déambule dans l'espace et fait le procès du Comte. Elle est invisible pour tous les personnages présents mais quand elle parle du Comte elle le regarde ou s'approche de lui. L'air adressé n'est plus seulement lyrique, il est aussi argumentatif. Lorsque la Comtesse sort par l'endroit où elle est arrivée, l'action du procès reprend sa vitesse normale. Au lieu de voir les personnages concernés entrer, on les voit finir une scène dans laquelle le Comte a été pleinement acteur et non plus seulement spectateur. Par ailleurs, grâce à la conjonction des deux procès, se sont superposées de façon très explicite la sphère privée et la sphère intime que le Comte a tendance à mêler de façon permanente. L'autre conséquence est de montrer une Comtesse plus active qu'elle ne l'est dans de nombreuses mises en scène. Elle ne compte pas seulement sur sa constance pour ramener le cœur infidèle, elle décide de passer à l'action. Le décalage entre son entrée un peu hésitante et sa sortie dynamique prouve que la confrontation fantasmatique à son mari lui a permis un affrontement qu'elle ne s'autorise pas dans la réalité.

L'entrée en scène d'un personnage qui vient chanter seul son air avant de repartir apparaît comme un condensé de conventions. En effet, tout est susceptible de paraître artificiel : la présence du personnage dans l'espace, le début et la fin de l'air, le déroulé du jeu privé d'adresse, le lien avec ce qui précède et ce qui suit, l'inscription de ce moment 
dans la logique de l'acte. C'est donc un problème dramaturgique qui ne doit pas être seulement appréhendé localement mais dans son lien avec un espace et un temps plus larges. En ce qui concerne Les Noces de Figaro, les metteurs en scène peuvent choisir de traiter l'entrée en scène de la Comtesse en mode mineur. Il s'agit alors essentiellement de gommer les dysfonctionnements pour centrer l'attention du spectateur sur un moment musical particulier. Le lyrisme, la psychologie, la beauté de l'air donnent à la scène la force d'une parenthèse dans le mouvement général de l'acte, au risque que la Comtesse paraisse en retrait par rapport à ce qui se joue en dehors d'elle. L'autre solution est au contraire de partir de l'évaluation des problèmes posés par cette entrée en scène pour en faire un moteur dramaturgique. En faisant entrer la Comtesse dans la scène du procès, Richard Brunel révèle une facette de l'air souvent ignorée, requestionne la place de l'épouse, fait de l'opéra avant tout une affaire de crise conjugale. Finalement, en refusant de nier ou de souligner la convention, en l'affrontant, il en fait un levier de sens, un instrument dramaturgique.

\section{NOTES}

1. Ces usages sont en train de disparaître au profit de la théâtralité. Ainsi, dans la mise en scène des Noces de Richard Brunel à laquelle nous consacrons la dernière partie de cet article, Mari Eriksmoen, la chanteuse qui jouait Barbarina, a accepté d'être présente sur la scène dès le début de l'opéra. Elle entre en scène avec le chœur, joue le rôle de la standardiste du Comte. On découvre qui elle est quand elle commence à chanter. Mais le spectateur l'a intégrée dans la fiction bien avant.

2. Il y a là un point de comparaison entre le boulevard et l'opéra. Le vedettariat y est encore mis en valeur. Au boulevard, on applaudit l'entrée en scène. À l'opéra, on applaudit la fin de l'air. Dans les deux cas, c'est un moment où le personnage s'efface derrière l'interprète. Parfois, ce dernier en joue en suspendant l'action, et en établissant avec le public une connivence extra fictionnelle.

3. La Finta giardiniera de Mozart est un modèle du genre. Les personnages entrent et sortent sans aucune justification dramaturgique.

4. On différenciera ce cas de celui où un personnage est laissé seul après une scène de groupe par exemple. La seule convention existante est alors celle du monologue chanté.

5. Da Ponte, pour Les Noces, montre, au début de trois actes sur quatre, un personnage seul. L'acte II commence par la cavatine de la Comtesse, l'acte III par un récitatif du Comte et l'acte IV par l'air de Barbarina.

6. Le chef est Jérémie Rhorer. Les chanteurs sont: Paolo Szot (le Comte), Malin Byström (la Comtesse), Patricia Petibon (Susanna), Kyle Ketelsen (Figaro), Kate Lindsey (Cherubino), Anna Maria Panzarella (Marcellina), Mario Luperi (Bartolo), John Graham-Hall (Basilio), Emanuele Giannino (Don Curzio), Mari Eriksmoen (Barberina), René Schirrer (Antonio).

7. Les mises en scène suivantes seront le étudiées dans cet article : Giorgio Strehler (1980), Peter Sellars (1990), Jean-Louis Martinoty (2004), Claus Guth (2006), Christoph Marthaler (2006), JeanFrançois Sivadier (2008), Sven-Eric Bechtolf (2009), Richard Brunel (2012). Le détail des équipes de création sera donné au fur et à mesure des références. 
8. Mozart, Les Noces de Figaro, L'Avant-scène Opéra, ${ }^{\circ} 21$, mai-juin 1979, p.78. Toutes les citations, ainsi que les traductions de Christiane Laurent, seront extraites de cet ouvrage.

9. La thématique judiciaire par exemple transforme l'espace en annexe d'une salle de procès. Le mariage, enjeu général de l'acte, ne suffit pas à l'unifier et donc à caractériser définitivement le lieu où sont les personnages.

10. Scène 6 (Marcellina, Bartolo, Figaro, Susanna), scène 7 (Barbarina, Cherubino), scène 8 (la Contessa), scène 9 (il Conte et Antonio), scène 10 (la Contessa, Susanna).

11. Pour la fin de la scène 7 et de la scène 9 . À la fin de la scène 8 , on a « elle part » et à la fin de la scène 6 « ils sortent ».

12. De fait dans cet acte, le hors-scène se révèle très présent dans le discours des personnages. Il existe un espace virtuel contigu dans lequel s'exerce la justice. C'est là que vont Figaro et Susanna à la fin de la troisième scène et c'est de là qu'arrivent tous les personnages concernés, à la fin de la cinquième scène. La Comtesse vient probablement de ses appartements. L'espace virtuel lointain est très présent aussi. Il concerne la maison d'Antonio dans laquelle Cherubino et Barbarina vont à la fin de la scène 7 et le jardin que Susanna, sur l'ordre de la Comtesse, a indiqué au Comte comme le lieu du rendez-vous.

13. Cette proposition est en réalité un ordre que la Comtesse a donné à Susanna au début de la scène 2 : « digli che ti attenda in giardino. » (dis-lui de t'attendre au jardin), op.cit, p.78.

14. "Et Suzanne qui ne vient pas! Je suis impatiente de savoir comment le Comte a accueilli sa proposition. Ce projet me semble très hardi ; c'est un époux si emporté et si jaloux... », op.cit, p. 90.

15. Le moment de l'entrée et la sortie de scène de Susanna n'est pas précisé dans le livret ce qui conduit là encore à un problème dramaturgique. En effet, si l'on s'appuie sur les événements, il est probable que Susanna assiste au verdict du procès et qu'elle sort pour aller chercher la bourse salvatrice. De fait, elle revient en ayant connaissance de l'alternative imposée par Curzio, à savoir payer ou épouser Marcellina. Or, L'Avant-scène Opéra, s'appuyant sur le fait qu'elle ne chante pas, ne l'intègre pas dans la liste des personnages du début de la scène 5 . On ne voit donc pas pourquoi elle reviendrait opportunément avec la somme d'argent, après être sortie à la scène 3 .

16. Op.cit., p.85.

17. Or, cela ne va pas de soi. En effet, Les Noces font partie des œuvres que l'on pourrait qualifier de peu ouvertes à l'audace et à l'innovation. Cela tient au statut de la comédie d'intrigue qui s'avère si contraignant que les espaces offerts aux écarts et à aux déplacements interprétatifs sont très limités.

18. Mise en scène présentée au Théâtre des Champs-Élysées en juin 2006. Le chef était René Jacobs. La distribution était composée de Pietro Spagnoli (le Comte), Annette Dasch (la Comtesse), Rosemary Joshua (Susanna), Luca Pisaroni (Figaro), Angelika Kirchschhlager (Cherubino), Sophie Pondjiclis (Marcellina), Alessandro Svab (Antonio), Antonio Abete (Bartolo), Enrico Facini (Basilio), Pauline Courtin (Barbarina), Serge Goubioud (Curzio). Captation BelAir Classiques, réalisée par Pierre Barré.

19. Parfois il crée, pour ce faire, des liaisons de scènes muettes. Ainsi, le duo de la lettre entre la Comtesse et Susanna est précédé d'une image rapide: celle de Marcellina, Bartolo, Figaro, en train de les quitter. Le metteur en scène prend au pied de la lettre ce que dit Susanna à la fin de la scène 6: «Voliamo ad informar d'ogni avventura Madama e nostro Zio.» (allons raconter ces aventures à Madame et à notre oncle), op.cit., p.88. La scène muette peut illustrer cela. Elle compense aussi le fait que Susanna ne donne aucune information sur le procès à la Comtesse. Martinoty comble là encore une lacune du livret puisque cette discussion n'a pas lieu ou du moins se déroule hors-scène.

20. Le spectacle a été capté à l'opéra de Zurich en 2009 pour EMI classics. Le chef était Franz Welser-Möst. Les chanteurs : Michael Volle (le Comte), Malin Hartelius (la Comtesse), Martina Jankovà (Susanna), Erwin Schrott (Figaro), Judith Schmid (Cherubino), Irène Friedli (Marcellina), 
Carlos Chausson (Bartolo), Martin Zysset (Basilio), Andreas Winkler (Curzio), Eva Liebau (Barbarina), Guiseppe Scorsin (Antonio).

21. Cette mise en scène date de 1980. Elle a été rejouée et captée en 2006 à la Scala et est disponible chez Arthaus Musik. Le chef était Gérard Korsten. On y trouve Pietro Spagnoli (le Comte), Marcella Orsatti Talamanca (la Comtesse), Diana Damrau (Susanna), Ildebrando d'Archangelo (Figaro), Monica Bacelli (Cherubino), Jeannette Fischer (Marcellina), Maurizio Muraro (Bartolo), Gregory Bonfatti (Basilio), Nicola Pamio (Curzio), Oriana Kurteshi (Barbarina), Matteo Peirone (Antonio).

22. Cette mise en scène a été enregistrée en1990. Elle est écoutable chez Decca. Le chef est Craig Smith. Les chanteurs sont James Maddalena (le Comte), Jayne West (la Comtesse), Jeanne Ommerlé (Susanna), Sanford Sylvan (Figaro), Susan Larson (Cherubino), Lynn Torgove (Barbarina), Frank Kelley (Don Basilio), David Evitts (Bartolo), Sue Ellen Kuzma (Marcellina), Hermann Hildebrand (Antonio), William Cotton (Curzio).

23. Sur cette scénographie d'Adrianne Lobel, voir: Maurin, Frédéric. Peter Sellars. Les Voies de la création théâtrale. Paris :CNRS éditions, 2003. P.36-39.

24. La captation faite par Arte en 2006 rassemble la distribution suivante: Peter Mattei (le comte), Christiane Oelze (la Comtesse), Heidi Grant Murphy (Susanna), Lorenzo Regazzo (Figaro), Christine Schäfer (Cherubino), Hélène Schneiderman (Marcellina), Roland Bracht (Bartolo), Burkhard Ulrich (Basilio), Eberhard Francesco Lorenz (Curzio), Cassandre Berthon (Barbarina), Frédéric Caton (Antonio).

25. Il s'agit là d'une production du Festival de Salzburg (2006) dirigée par Nikolaus Harnoncourt avec Bo Skovhus (le Comte), Dorothea Röschmann (la Comtesse), Ildebrando d'Arcangelo (Figaro), Anna Netrebko (Susanna), Christine Schäfer (Cherubino), Marie Mac Laughlin (Marcellina), Franz-Josef Selig (Bartolo), Patrick Henckens, Oliver Ringelhahn (Curzio), Florian Boesch (Antonio), Eva Liebau (Barbarina), Uli Kirsch (Cherubino).

26. Relevant de la science quand ils sont manipulés par le Comte, les objets se transforment en emblèmes des tableaux de vanité quand ils sont tenus par la Comtesse.

27. La collection est sans doute aussi une référence au XVIII ${ }^{\text {ème }}$ siècle.

28. Dans sa mise en scène de 2008. L'orchestre était dirigé par Emmanuelle Haïm. Les interprètes: Jacques Imbrailo (le Comte), Nicole Heaston (la Comtesse), Matthew Rose(Figaro), Hélène Guilmette (Susanna ), Kate Lindsay (Cherubino), Paolo Battaglia (Bartolo), Anne Mason (Marcellina), Cyril Auvity (Basilio), Carl Ghazarossian (Curzio), Hanna Bayodi-Hirt (Barbarina), Christian Tréguier (Antonio). Jean-François Sivadier est un metteur en scène d'opéra reconnu. Il a aussi écrit une pièce en 1996, Italienne avec orchestre, dans laquelle il décrit les coulisses d'une répétition. La question de l'entrée en scène y est envisagée.

29. Notons d'ailleurs que Strehler intègre les applaudissements du public dans ce cheminement. Avant de quitter l'espace, la Comtesse s'appuie contre le mur ce qui permet à la chanteuse de recevoir l'hommage des spectateurs. Néanmoins, elle est placée de dos ce qui intègre ce geste à la mise en scène et évite le pire, c'est-à-dire la sortie ponctuelle du rôle. Il n'est pas rare aujourd'hui que les metteurs en scène enchaînent directement l'action après l'air. Les applaudissements se produisent alors dans une sorte de vide alors que le chanteur est sorti ou que l'on est clairement passé à autre chose.

30. Cette innovation a été modérément appréciée par les tenants du respect de l'œuvre!

31. Le spectateur peut alors faire vivre par l'imagination une conversation qui a lieu hors-scène et qui se poursuit devant nous. La Comtesse, se plaignant de ne pas pouvoir trouver Susanna, est allée la chercher et la ramène. Cette micro séquence cohérente vient compenser rétrospectivement l'incohérence de la place de l'air.

32. On peut noter aussi le même effort que chez Strehler pour rendre cohérent le lien entre l'air de la Comtesse et ce qui suit. L'entrée en scène de la Comtesse n'est pas forcément logiquement placée avec ce qui précède mais elle l'est avec les scènes à venir. 
33. Marthaler pendant l'air de la Comtesse fait passer des mariés dans le bureau d'état-civil. Mais cela n'agit pas avec la scène principale. C'est un second plan qui a une fonction de décor.

34. Mais ces appuis de jeu sont abandonnés ensuite pour laisser la place à des mouvements du corps. La Comtesse suit le rythme de la musique pour improviser une marelle. Ce faisant, elle redevient la Rosine qu'elle avait cessé d'être en épousant le Comte.

35. Dans les mises en scène de Jean-François Sivadier la perruque est une sorte de masque émotionnel. Le personnage l'enlève à un moment de mutation ou à un moment de vérité.

36. L'abandon des récitatifs originaux au profit d'un retour de l'orchestre est comme une signature de ce classicisme comme si face à la beauté de l'air, la mise en scène renonçait à l'originalité.

37. Il faudrait aussi s'interroger sur le costume du personnage à ce moment précis. Dans la majorité des mises en scène, la Comtesse entre en scène en robe de soirée comme si elle était prête à assister au mariage. Or, à ce stade comment peut-elle savoir que le mariage aura lieu de façon certaine si elle n'a pas revu Suzanne?

38. Dans sa trilogie sur Kafka (2000), il y avait déjà l'air de Barbarina.

39. Dans L'Opéra des gueux, palimpseste pour une fin de siècle de John Gay (1996).

40. Comme Isabelle Bonnadier dans Kasimir et Caroline de Horvath (2002).

41. Pour Au bord, spectacle sur Maupassant.

42. Der Jasager de Brecht et Weill (2006), Infedeltà delusa de Haydn (2008), In the penal colony de Philip Glass ((2009), Albert Herring de Britten (2009), Lakmé de Delibes (2009), Re Orso de Marco Stroppa (2012), Der kaiser von Atlantis de Ullmann.

43. Ce débat est encore très vivace comme en témoigné les articles de presse portant sur ce spectacle.

44. Le résultat est tel que le spectateur peut goûter le spectacle et écouter la musique sans avoir recours aux surtitres.

45. Travail auquel est évidemment associé le ou la dramaturge.

46. Contrairement à ce qui se passe au théâtre, les contraintes spécifiques de l'opéra obligent à imaginer la mise en scène sur le papier. Le temps de répétition est en effet très court pour des œuvres d'une telle complexité et il est inimaginable d'arriver sur une production sans avoir une idée très claire de ce que l'on cherche. Évidemment, le travail avec les chanteurs modifie parfois considérablement l'imaginaire préalable.

47. Alors que jusque-là elle agissait par procuration ou en secret, elle décide à ce moment qu'elle prendra les choses en main même quand plus rien ne fait obstacle au mariage de Susanna. Se dessine alors une nouvelle facette du personnage pour qui la fin justifie les moyens.

48. Dans le travail de Richard Brunel, la place du public est toujours centrale. Elle commence par la façon dont le spectateur connecte ce qu'il vit et ce qu'il voit. Ainsi, en assistant aux Noces de Figaro, quel lien fait-il avec aujourd'hui qui lui fait entendre l'opéra de façon particulière, dans une résonnance à la fois intime et politique ? Les connexions avec des événements récents et en particulier dans des affaires qui mêlent le monde du pouvoir avec le sexe étaient possibles.

49. Le mot « justice » est très présent dans l'opéra.

50. « Lo son qui per giudicar » (je suis là pour rendre justice), scène 12 de l'acte II, op.cit, p.71.

51. Il recommence plus tard. L'acte IV commence en effet par une scène de banquet exclusivement théâtrale avec du texte. La musique ne reprend qu'avec l'air de Barbarina. Une fois de plus se joue une sorte de conflit entre l'opéra et le théâtre, entre la musique et le silence. 52. « Ma che mal c'è ?» (mais quel mal y a-t-il ?), op.cit, p.90.

53. « a quale/ umil stato fatale io son ridotta/ da un consorte crudel, che dopo avermi/ con un misto inaudito/ d'infedeltà, di gelosia, di sdegno,/ prima amata, indi offesa, e alfin tradita,/ fammi or cercar da una mia serva aita ! »... » (à quelle humiliante situation suis-je réduite par ce mari cruel qui, après m'avoir, par un mélange inouï d'infidélités, de jalousie et de dédain, d'abord aimée puis offensée, enfin trahie, me contraint à rechercher l'aide de ma servante !), Ibid. 


\section{RÉSUMÉS}

L'entrée en scène à l'opéra pose des questions spécifiques et s'avère souvent plus contraignante qu'au théâtre. Néanmoins, elle peut être l'objet d'un traitement particulier, d'un geste appuyé du metteur en scène. L'article s'intéresse à l'entrée en scène de la Comtesse Almaviva dans la scène 8 de l'acte III des Noces de Figaro. S'appuyant principalement sur la mise en scène de Richard Brunel, présentée au Festival d'Aix en Provence en 2012, il parcourt tout le cheminement dramaturgique qui a mené au plateau: étude des problèmes posés par cette entrée en scène, analyse d'autres mises en scène, choix opérés.

\section{INDEX}

Mots-clés : Beaumarchais, opéra, dramaturgie, Brunel (Richard)

\section{AUTEUR}

\section{CATHERINE AILLOUD-NICOLAS}

Maître de conférences, agrégée de Lettres Modernes, membre de l'UMR LIRE, Catherine AilloudNicolas est spécialiste de Marivaux et de la dramaturgie classique. Ses recherches portent sur la représentation théâtrale le passage du texte à la scène. Elle enseigne à l'Université Lyon I (IUFM) et est professeur de dramaturgie dans la classe de théâtre du Conservatoire de Lyon. Dramaturge pour le théâtre, l'opéra, la danse et les spectacles de marionnette, elle a collaboré avec Eric Massé (L'Île des esclaves, Macbeth), Hervé Datiguelongue (Les Précieuses ridicules, Amphitryon de Plaute), Johanny Bert (L'Opéra de quat'sous). Elle travaille régulièrement avec Richard Brunel pour le théâtre (Hedda Gabler) et pour l'opéra (L'infedeltà delusa de Haydn, Lakmé de Delibes, La Colonie pénitentiaire de Glass, Les Noces de Figaro de Mozart, et le Re Orso de Marco Stroppa dont elle a coécrit le livret. Elle est également membre du collectif artistique de la Comédie de Valence - CDN Drôme-Ardèche, dirigé par Richard Brunel depuis janvier 2010. 